Homology, Homotopy and Applications, vol.19(1), 2017, pp.209-224

\title{
POSTNIKOV DECOMPOSITION AND THE GROUP OF SELF-EQUIVALENCES OF A RATIONALIZED SPACE
}

\author{
MAHMOUD BENKHALIFA
}

(communicated by Alexander Mishchenko)

\begin{abstract}
Let $X$ be a simply connected rational CW complex of finite type. Write $X^{[n]}$ for the $n$th Postnikov section of $X$. Let $\mathcal{E}\left(X^{[n+1]}\right)$ denote the group of homotopy self-equivalences of $X^{[n+1]}$. We use Sullivan models in rational homotopy theory to construct two short exact sequences:

$$
\operatorname{Hom}\left(\pi_{n+1}(X) ; H^{n+1}\left(X^{[n]}\right)\right) \longmapsto \mathcal{E}\left(X^{[n+1]}\right) \rightarrow D_{n}^{n+1},
$$$$
\operatorname{Hom}\left(\pi_{n+1}(X) ; H^{n+1}\left(X^{[n]}\right)\right) \longmapsto \mathcal{E}_{\sharp}\left(X^{[n+1]}\right) \rightarrow G_{n}^{n+1},
$$

where $D_{n}^{n+1}$ is a subgroup of $\operatorname{aut}\left(\operatorname{Hom}\left(\pi_{q}(X) ; \mathbb{Q}\right)\right) \times \mathcal{E}\left(X^{[n]}\right)$ which is defined in terms of the Whitehead exact sequence of $X$ and where $G_{n}^{n+1}$ is a certain subgroup of $\mathcal{E}_{\sharp}\left(X^{[n]}\right)$. Here $\mathcal{E}_{\sharp}\left(X^{[n]}\right)$ is the subgroup of those elements inducing the identity on the homotopy groups. Moreover, we give an alternative proof of the Costoya-Viruel theorem [9]: Every finite group occurs as $\mathcal{E}(X)$ where $X$ is rational.
\end{abstract}

\section{Introduction}

Let $\mathcal{E}(X)$ denote the group of self homotopy equivalences of a simply connected $\mathrm{CW}$-complex $X$ and let $\mathcal{E}_{\sharp}(X)$ denote the subgroup represented by self-equivalences that induce the identity map on $\pi_{*}(X)$. The subgroup $\mathcal{E}_{\sharp}(X)$ is not in general trivial, for instance in [3] it is shown that $\mathcal{E}_{\sharp}\left(S^{2} \times S^{n}\right) \cong \pi_{n+2}\left(S^{2}\right) \oplus \mathbb{Z}_{2}$ where $n \geqslant 3$.

The study of the groups $\mathcal{E}(X)$ and $\mathcal{E}_{\sharp}(X)$ by means of a cellular decomposition of $X$ is a difficult problem with a long history. See Rutter [11, Chapter 11] for a survey.

When $X$ is a simply connected rational CW complex of finite type, i.e., $\pi_{n}(X)$ is a vector space of finite dimension for every $n \geqslant 1$, the group $\mathcal{E}(X)$ has emerged as a recent object of interest, for instance the realization problem, namely which group occurs as $\mathcal{E}(X)$ ? Arkowitz-Lupton [2] gave the first examples of finite groups occurring as $\mathcal{E}(X)$. Further examples were given by the author in $[\mathbf{6}, \mathbf{7}]$. CostoyaViruel [9] then proved the remarkable result that every finite group $G$ occurs as $G=\mathcal{E}(X)$ for some elliptic rational space $X$. All these works have been accomplished

Received January 14, 2016, revised May 16, 2016; published on March 29, 2017.

2010 Mathematics Subject Classification: 55P10.

Key words and phrases: group of homotopy self-equivalences, Whitehead exact sequence, Sullivan model, rational homotopy theory, Postnikov tower.

Article available at http://dx.doi.org/10.4310/HHA.2017.v19.n1.a11

Copyright (C) 2017, International Press. Permission to copy for private use granted. 
using Sullivan models in rational homotopy theory. A similar approach of studying the group $\mathcal{E}(X)$ based on Anick's DG Lie algebra models [1] over a certain subring of $\mathbb{Q}$ has been developed by Benkhalifa-Smith [8].

The aim of this paper is to study the effect of rational cell-attachment on the group of self-equivalences using the Postnikov tower. More precisely let $X$ be a simply connected rational CW complex of finite type. Write $X^{[n]}$ for the $n$th Postnikov section of $X$ [4, page 237]. We consider the situation in which

$$
X^{[n]} \cup_{\alpha}\left(\bigcup_{i \in I} e_{i}^{q}\right), \quad \text { where } I \text { is finite and } q>n,
$$

is the space obtained by attaching rational cells to $X^{[n]}$ by a map $\alpha: \underset{i \in I}{\vee} S_{\mathbb{Q}}^{q} \rightarrow X^{[n]}$ where $S_{\mathbb{Q}}^{q}$ is the rational sphere of dimension $q\left[\mathbf{1 0}\right.$, page 102]. Let $Z^{q}$ be the pullback of the map $X^{[n]} \rightarrow K\left(\pi_{q}(X) ; q+1\right)$ over the path fibration:

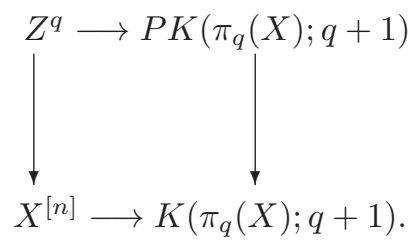

Recall that if $q=n+1$, then the space $Z^{q}$ coincides with $(n+1)$ th Postnikov section $X^{[n+1]}$ of $X$.

We prove:

Theorem 1.1. There exist two short exact sequences of groups:

$$
\begin{aligned}
\operatorname{Hom}\left(\pi_{q}(X) ; H^{n+1}\left(X^{[n]}\right)\right) & \longmapsto \mathcal{E}\left(Z^{q}\right) \rightarrow D_{n}^{q}, \\
\operatorname{Hom}\left(\pi_{q}(X) ; H^{n+1}\left(X^{[n]}\right)\right) & \longmapsto \mathcal{E}_{\sharp}\left(Z^{q}\right) \rightarrow G_{n}^{q},
\end{aligned}
$$

where $D_{n}^{q}$ (respect. $\left.G_{n}^{q}\right)$ is a certain subgroup of $\operatorname{aut}\left(\operatorname{Hom}\left(\pi_{q}(X) ; \mathbb{Q}\right)\right) \times \mathcal{E}\left(X^{[n]}\right)($ respect. of $\mathcal{E}_{\sharp}\left(X^{[n]}\right)$ ) (see (14) and (15) for the definitions).

Consequently, we prove the following first result concerning the question of (in)finiteness of the group $\mathcal{E}\left(X^{[n]}\right)$.

Corollary 1.2. Let $X$ be a simply connected rational $C W$ complex of finite type. Assume that $\pi_{i}(X) \otimes \mathbb{Q} \neq 0$ for $i=n-1, n, n+1$.

If $\mathcal{E}\left(X^{[n]}\right)$ is finite, then $\mathcal{E}\left(X^{[n+1]}\right)$ and $\mathcal{E}\left(X^{[n-1]}\right)$ are infinite.

Next we prove the following result showing the relationship between the finiteness of the group $\mathcal{E}\left(Z^{n+2}\right)$ and the Postnikov invariant $\left[k^{n}\right]$ of the space $X$ [4, page 237].

Corollary 1.3. Let $X$ be a simply connected rational $C W$ complex of finite type. Assume that $\mathcal{E}\left(Z^{n+2}\right)$ is finite, then the space $X^{[n+1]}$ has the homotopy type of $X^{[n]} \times$ $K\left(\pi_{n+1}(X), n+1\right)$.

In addition we give a second proof of Costoya-Viruel in [9] that every finite group occurs as $\mathcal{E}(X)$ for some elliptic rational space $X$.

We establish these results in an algebraic context using the notion of Sullivan models in rational homotopy theory [12]. Recall that if $X$ is a simply connected 
rational $\mathrm{CW}$ complex of finite type, then there exists a free commutative cochain algebra $(\Lambda V, \partial)$ called the Sullivan model of $X$, unique up to isomorphism, which determines completely the homotopy type of the space $X$. Moreover, the Sullivan model recovers homotopy data via the identifications:

$$
\operatorname{Hom}\left(\pi_{*}(X), \mathbb{Q}\right) \cong V^{*}, \quad H^{*}(X ; \mathbb{Q}) \cong H^{*}(\Lambda V, \partial) \quad \text { and } \quad \mathcal{E}(X) \cong \frac{\operatorname{aut}(\Lambda V, \partial)}{\simeq},
$$

where $\frac{\operatorname{aut}(\Lambda V, \partial)}{\simeq}$ is the group of homotopy cochain self-equivalences of $(\Lambda V, \partial)$ modulo the relation of homotopy between free commutative cochain algebras (see $[\mathbf{1 2}]$ ). We write

$$
\mathcal{E}(\Lambda V)=\operatorname{aut}(\Lambda V, \partial) / \simeq,
$$

for this group. Similarly, we have $\mathcal{E}_{\sharp}(X) \cong \operatorname{aut}_{\sharp}(\Lambda V, \partial) / \simeq$. We denote the latter group by $\mathcal{E}_{\sharp}(\Lambda V)$. Here $\operatorname{aut}_{\sharp}(\Lambda V, \partial)$ is the group of homotopy cochain self-equivalences inducing the identity automorphism on $V^{*}$.

The exact sequences in Theorem 1.1 are then the translation of the exact sequences given, in (11), for $\mathcal{E}(\Lambda V)$ and $\mathcal{E}_{\sharp}(\Lambda V)$. We end this work by giving an example showing that there exists a free commutative cochain algebra $(\Lambda V, \partial)$, which is not of finite type, such that $\mathcal{E}(\Lambda V) \cong \mathbb{Z}$.

\section{Homotopy self-equivalences of cochain morphisms}

\subsection{Notation and fundamental results}

All vector spaces, algebras, tensor products, etc. are defined over $\mathbb{Q}$ and this ground field will be in general suppressed from the notation.

A commutative cochain algebra is a (positive) graded differential vector space equipped with a graded linear map $\partial^{*}$ (called the differential) $\left(\partial^{n}: A^{n} \rightarrow A^{n+1}\right.$, such that $\left.\partial^{2}=0\right)$ together with linear maps $A^{*} \otimes A^{*} \rightarrow A^{*}$, denoted by $(a \otimes b \rightarrow a . b)$ and $i: \mathbb{Q} \rightarrow A^{*}$ which satisfy the associativity, commutativity (in the graded sense), and unit conditions and the following relations:

$$
A^{i} \cdot A^{j} \subset A^{i+j} \quad \text { and } \quad d(a . b)=d(a) \cdot b+(-1)^{|a|} a . d(b) \quad, \quad a, b \in A .
$$

A commutative cochain algebra $\left(A^{*}, d\right)$ is said to be 1 -connected if $H^{0}(A)=\mathbb{Q}$ and $H^{1}(A)=0$, and of finite type if each vector space $H^{n}(A)$ is finite dimensional.

Definition 2.1. Let $V$ be a (positive) graded vector space and let $T(V)$ be the free tensor algebra over $V$. Put $T(V)=\mathbb{Q} \oplus T^{\geqslant 1}(V)$ and define $\Lambda V$, called the free commutative algebra, to be $T(V)$ divided by the two-sided ideal generated by elements of the form (a.b).c-a.(b.c) and $a . b-(-1)^{|a||b|} b . a$ where $a, b, c \in T(V)$.

The free commutative algebra $\Lambda V$ can also be described as follows: $\Lambda V=E\left(V^{\text {odd }}\right) \otimes$ $S\left(V^{\text {even }}\right)$, where $E\left(V^{\text {odd }}\right)$ is the exterior algebra on the oddly graded part of $V$ and $S\left(V^{\text {even }}\right)$ is the symmetric algebra on the evenly graded part of $V$. Moreover, if $\partial$ is a differential on $\Lambda V$, then $(\Lambda V, \partial)$ is called a free commutative cochain algebra (fcca for short).

Let us recall the following results (see $[\mathbf{1 0}, \mathbf{1 2}]$ ) which are fundamental in rational homotopy theory. 
Theorem 2.2. For every 1-connected commutative cochain algebra $\left(A^{*}, d\right)$, there exists a quasi-isomorphism $(\Lambda V, \partial) \rightarrow\left(A^{*}, d\right)$ such that $\partial$ is decomposable, i.e., $\operatorname{Im} \partial \subset$ $\Lambda^{+} V . \Lambda^{+} V$. The $f c c a(\Lambda V, \partial)$ is called a minimal Sullivan model of $\left(A^{*}, d\right)$, it is unique up to isomorphism. Moreover, two 1-connected commutative cochain algebras are quasi-isomorphic if and only if their minimal Sullivan models are isomorphic.

Theorem 2.3. Let $X$ be a simply connected $C W$-complex having rational homology of finite type. Let $A_{P L}(X)$ be the simplicial cochain algebra associated with $X$ (see $[10,12])$. The minimal Sullivan model $(\Lambda V, \partial)$ of $A_{P L}(X)$ is called the minimal Sullivan model of $X$. Recall that $H^{*}(\Lambda V, \partial) \cong H^{*}(X, \mathbb{Q})$, as graded algebras, and $V^{n} \cong \operatorname{Hom}_{\mathbb{Z}}\left(\pi_{n}(X) ; \mathbb{Q}\right)$ for every $n \geqslant 2$. In addition there exists a bijection between the set of rational homotopy types of simply connected $C W$-complexes having rational homology of finite type and the set of isomorphism classes of fccas.

\subsection{Notion of homotopy for free commutative cochain algebras}

Definition 2.4. Let $(\Lambda(V), \partial)$ be a 1-connected fcca. Define the vector spaces $\bar{V}$ and $\widehat{V}$ by $(\bar{V})^{n}=V^{n+1}$ and $(\widehat{V})^{n}=V^{n}$. We then define the fcca $(\Lambda(V, \bar{V}, \widehat{V}), D)$ with the differential $D$ is given by

$$
D(v)=\partial(v), \quad D(\widehat{v})=0, \quad D(\bar{v})=\widehat{v} .
$$

We define a derivation $S$ of degree -1 of the fcca $(\Lambda(V, \bar{V}, \widehat{V}), D)$ by putting $S(v)=\bar{v}$, $S(\bar{v})=S(\widehat{v})=0$.

A homotopy between two cochain morphisms $\alpha, \alpha^{\prime}:(\Lambda(V), \partial) \rightarrow(\Lambda(V), \partial)$ is a cochain morphism

$$
F:(\Lambda(V, \bar{V}, \widehat{V}), D) \rightarrow(\Lambda(V), \partial),
$$

such as $F(v)=\alpha(v)$ and $F \circ e^{\theta}(v)=\alpha^{\prime}(v)$, where

$$
e^{\theta}(v)=v+\widehat{v}+\sum_{n \geqslant 1} \frac{1}{n !}(S \circ \partial)^{n}(v), v \in V \quad \text { and } \quad \theta=D \circ S+S \circ D \text {. }
$$

Thereafter we will need the following lemma:

Lemma 2.5. Let $q>n$ and let $V=V^{q} \oplus V^{\leqslant n}$ and $\alpha, \alpha^{\prime}:(\Lambda(V), \partial) \rightarrow(\Lambda(V), \partial)$ be two cochain morphisms satisfying:

$$
\alpha(v)=v+z, \quad \alpha(v)=v+z^{\prime} \text { on } V^{q} \quad \text { and } \quad \alpha=\alpha^{\prime}=\text { id } \quad \text { on } V^{\leqslant n} .
$$

Assume that $z-z^{\prime}=\partial(u)$, where $u \in \Lambda(V)$. Then $\alpha$ and $\alpha^{\prime}$ are homotopic.

Proof. Define $F$ by setting

$$
\begin{aligned}
& F(v)=v+y, \quad F(\widehat{v})=z^{\prime}-z \quad \text { and } \quad F(\bar{v})=z \quad \text { for } \quad v \in V^{q}, \\
& F(v)=v, \quad F(\widehat{v})=0 \quad \text { and } \quad F(\bar{v})=0 \quad \text { for } \quad v \in V^{\leqslant n}
\end{aligned}
$$

then $F$ is the needed homotopy. 


\subsection{The graded linear map $b^{*}$}

Definition 2.6. Let $\left(\Lambda\left(V^{q} \oplus V^{\leqslant n}\right), \partial\right)$ be a 1-connected fcca where $q>n$. We define the linear map $b^{q}: V^{q} \rightarrow H^{q+1}\left(\Lambda V^{\leqslant n}\right)$ by setting

$$
b^{q}(v)=[\partial(v)], \quad v \in V^{q} .
$$

Here $[\partial(v)]$ denotes the cohomology class of $\partial(v) \in\left(\Lambda V^{\leqslant n}\right)^{q+1}$.

For every 1-connected cdga $\left(\Lambda\left(V^{q} \oplus V^{\leqslant n}\right), \partial\right)$, the linear map $b^{q}$ is natural. Namely, if $[\alpha] \in \mathcal{E}\left(\Lambda\left(V^{q} \oplus V^{\leqslant n}\right)\right)$, then the following diagram commutes:

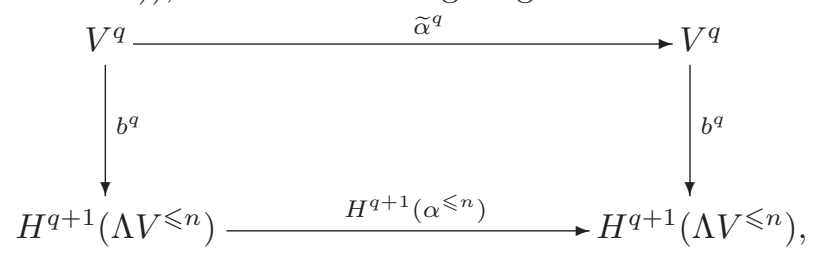

where $\widetilde{\alpha}: V^{*} \rightarrow V^{*}$ is the graded homomorphism induced by $\alpha$ on the indecomposables and where $\alpha^{\leqslant n}:\left(\Lambda V^{\leqslant n}, \partial\right) \rightarrow\left(\Lambda V^{\leqslant n}, \partial\right)$ is the restriction of $\alpha$.

\subsection{The groups $\mathcal{D}_{n}^{q}$}

Definition 2.7. Given a 1-connected fcca $\left(\Lambda\left(V^{q} \oplus V^{\leqslant n}\right), \partial\right)$ where $q \geqslant n$, let $\mathcal{D}_{n}^{q}$ be the subset of aut $\left(V^{q}\right) \times \mathcal{E}\left(\Lambda V^{\leqslant n}\right)$ consisting of the couples $\left(\xi,\left[\alpha^{\leqslant n}\right]\right)$ making the following diagram commute:

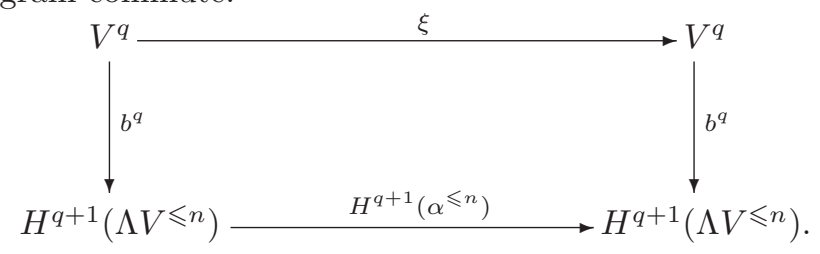

Clearly, $\mathcal{D}_{n}^{q}$ is a subgroup of $\operatorname{aut}\left(V^{q}\right) \times \mathcal{E}\left(\Lambda V^{\leqslant n}\right)$.

Proposition 2.8. The map g: $\mathcal{E}\left(\Lambda\left(V^{q} \oplus V^{\leqslant n}\right)\right) \rightarrow \mathcal{D}^{q}$ given by

$$
\mathrm{g}([\alpha])=\left(\widetilde{\alpha}^{q},\left[\alpha^{\leqslant n}\right]\right)
$$

is a surjective homomorphism of groups.

Proof. First it is well-known [10, Proposition 12.8] that if two cochain morphisms are homotopic, then they induce the same graded linear maps on the indecomposables, i.e., $\widetilde{\alpha}=\widetilde{\alpha^{\prime}}$, moreover, $\alpha^{\leqslant n}, \alpha^{\prime \leqslant n}$ are homotopic and by using the diagram (3) we deduce that the map $\mathrm{g}$ is well-defined.

Next let $\left(\xi,\left[\alpha^{\leqslant n}\right]\right) \in \mathcal{D}_{n}^{q}$. Recall that, in the diagram (4), we have:

$$
\begin{aligned}
& H^{q+1}\left(\alpha^{\leqslant n}\right) \circ b^{q}(v)=\alpha^{\leqslant n} \circ \partial(v)+\operatorname{Im} \partial^{\leqslant n}, \\
& b^{q} \circ \xi^{q}(v)=\partial \circ \xi^{q}(v)+\operatorname{Im} \partial^{\leqslant n},
\end{aligned}
$$

where $\partial^{\leqslant n}:\left(\Lambda V^{\leqslant n}\right)^{q} \rightarrow\left(\Lambda V^{\leqslant n}\right)^{q+1}$.

Since by Definition 2.7 this diagram commutes, the element $\left(\alpha^{\leqslant n} \circ \partial-\partial \circ \xi^{q}\right)(v) \in$ $\operatorname{Im} \partial^{\leqslant n}$. As a consequence there exists $u_{v} \in\left(\Lambda V^{\leqslant n}\right)^{q}$ such that

$$
\left(\alpha^{\leqslant n} \circ \partial-\partial \circ \xi^{q}\right)(v)=\partial^{\leqslant n}\left(u_{v}\right) .
$$


Thus we define $\alpha:\left(\Lambda\left(V^{q} \oplus V^{\leqslant n}\right), \partial\right) \rightarrow\left(\Lambda\left(V^{q} \oplus V^{\leqslant n}\right), \partial\right)$ by setting

$$
\alpha(v)=\xi^{q}(v)+u_{v}, \quad \text { and } \quad \alpha=\alpha^{\leqslant n} \text { on } V^{\leqslant n} .
$$

As $\partial(v) \in\left(\Lambda V^{\leqslant n}\right)^{q}$ then, by (6), we get

$$
\partial \circ \alpha(v)=\partial\left(\xi^{q}(v)\right)+\partial^{\leqslant n}\left(u_{v}\right)=\alpha^{\leqslant n} \circ \partial(v)=\alpha \circ \partial(v) .
$$

So $\alpha$ is a cochain morphism. Now due to the fact that $u_{v} \in\left(\Lambda V^{\leqslant n}\right)^{q}$ and $q>n$, the linear map $\widetilde{\alpha}^{q}: V^{q} \rightarrow V^{q}$ coincides with $\xi^{q}$.

Then it is well-known (see [10]) that any cochain morphism between two 1connected fccas inducing a graded isomorphism on the indecomposables is a homotopy equivalence. Consequently, $[\alpha] \in \mathcal{E}(\Lambda V, \partial)$. Therefore $\mathrm{g}$ is surjective.

Finally, the following relations:

$$
\begin{aligned}
\mathrm{g}\left([\alpha]\left[\alpha^{\prime}\right]\right) & =\mathrm{g}\left(\left[\alpha \circ \alpha^{\prime}\right]\right)=\left({\widetilde{\alpha \circ \alpha^{\prime}}}^{q},\left[\alpha^{\leqslant n} \circ \alpha^{\prime \leqslant n}\right]\right) \\
& =\left(\widetilde{\alpha}^{q},\left[\alpha^{\leqslant n}\right]\right) \circ\left({\widetilde{\alpha^{\prime}}}^{q},\left[\alpha^{\prime \leqslant n}\right]\right)=\mathrm{g}([\alpha]) \circ \mathrm{g}\left(\left[\alpha^{\prime}\right]\right)
\end{aligned}
$$

assure that $\mathrm{g}$ is a homomorphism of groups.

\subsection{Characterization of ker g}

Next by definition we have

$$
\operatorname{ker} g=\left\{[\alpha] \in \mathcal{E}(\Lambda(V)) \mid \widetilde{\alpha}^{q}=i d_{V^{q}}, \quad\left[\alpha^{\leqslant n}\right]=\left[i d_{\Lambda(V \leqslant n}\right]\right\},
$$

therefore for every $[\alpha] \in \operatorname{ker} g$ we have

$$
\begin{aligned}
& \alpha(v)=v+z, \quad z \in \Lambda^{q}\left(V^{\leqslant n}\right), \\
& \alpha^{\leqslant n} \simeq i d_{\Lambda(V \leqslant n) .}
\end{aligned}
$$

So define

$$
\theta_{\alpha}: V^{q} \rightarrow \Lambda^{q}\left(V^{\leqslant n}\right) \quad \text { by } \quad \theta_{\alpha}(v)=\alpha(v)-v .
$$

Notice that the relations (7) and (8) imply that

$$
\theta_{\alpha^{\prime} \circ \alpha}=\theta_{\alpha^{\prime}}+\theta_{\alpha} .
$$

Lemma 2.9. Let $[\alpha] \in$ ker g. Then there exists $[\beta] \in$ ker g satisfying:

1. $\theta_{\beta}(v)$ is a cocycle in $\Lambda^{q}\left(V^{\leqslant n}\right)$ for every $v \in V^{q}$

2. $\beta_{\leqslant n}=\alpha \leqslant n$

3. $[\beta]=[\alpha]$

Proof. Since $\left[\alpha^{\leqslant n}\right]=\left[i d_{\Lambda(V \leqslant n)}\right]$ there is a homotopy

$$
F:\left(\Lambda\left(V^{\leqslant n}, \bar{V}^{\leqslant n}, \widehat{V}^{\leqslant n}\right), D\right) \rightarrow\left(\Lambda\left(V^{\leqslant n}\right), \partial\right),
$$

such that $F(v)=v$ and $F \circ e^{\theta}(v)=\alpha^{\leqslant n}(v)$. Therefore for $v \in V^{q}$ the element $F\left(\sum_{n \geqslant 1} \frac{1}{n !}(S \circ \partial)^{n}(v)\right)$ is a well-defined element in $\Lambda^{q}\left(V^{\leqslant n}\right)$. Thus we define $\beta$ by setting

$$
\beta(v)= \begin{cases}v, & \text { for } v \in V^{q} \\ \alpha(v)-F\left(\sum_{n \geqslant 1} \frac{1}{n !}(S \circ \partial)^{n}(v)\right), & \text { for } v \in V^{\leqslant n}\end{cases}
$$


Given $v \in V^{q}$ we compute

$$
\begin{aligned}
\partial\left(\theta_{\beta}(v)\right) & =\partial\left(\alpha(v)-F\left(\sum_{n \geqslant 1} \frac{1}{n !}(S \circ \partial)^{n}(v)\right)-v\right) \\
& =\alpha(\partial(v))-\alpha \circ F\left(\sum_{n \geqslant 1} \frac{1}{n !}(S \circ \partial)^{n}(v)\right)-\partial(v) \\
& =F \circ e^{\theta}(\partial(v))-F \circ D\left(\sum_{n \geqslant 1} \frac{1}{n !}(S \circ \partial)^{n}(v)\right)-\partial(v) \\
& =F \circ D \circ e^{\theta}(v)-F \circ D\left(\sum_{n \geqslant 1} \frac{1}{n !}(S \circ \partial)^{n}(v)\right)-\partial(v) \\
& =F \circ D(v+\widehat{v})-\partial(v) \\
& =\partial(v)-\partial(v)=0 .
\end{aligned}
$$

Thus $\beta$ satisfies (1). For (2) and (3), we define $G:(\Lambda(V, \bar{V}, \widehat{V}), D) \rightarrow(\Lambda(V), \partial)$ by setting $G=F$ on $\left(\Lambda\left(V^{\leqslant n}, \bar{V}^{\leqslant n}, \widehat{V}^{\leqslant n}\right), D\right)$ while, for $v \in V^{q}$, we set $G(v)=\beta(v)$ and $G(\widehat{v})=G(\bar{v})=0$. First, it is easy to check that $G$ is a cochain morphism. Next, for $v \in V^{q}$ we have

$$
\begin{aligned}
G \circ e^{\theta}(v) & =G\left(v+\widehat{v}+\sum_{n \geqslant 1} \frac{1}{n !}(S \circ \partial)^{n}(v)\right)=G(v)+G\left(\sum_{n \geqslant 1} \frac{1}{n !}(S \circ \partial)^{n}(v)\right) \\
& =\beta(v)+F\left(\sum_{n \geqslant 1} \frac{1}{n !}(S \circ \partial)^{n}(v)\right)=\alpha(v) .
\end{aligned}
$$

Therefore $\beta \simeq \alpha$ and the lemma is proved.

Thus Lemma 2.9 and the relation (8) allow us to define a map $\Phi$ : ker $\mathrm{g} \rightarrow$ $\operatorname{Hom}\left(V^{q}, H^{q}\left(\Lambda\left(V^{\leqslant n}\right)\right)\right)$ by setting $\Phi([\beta])(v)=\left\{\theta_{\beta}(v)\right\}$ for $v \in V^{q}$ where $[\beta]$ is chosen as in Lemma 2.9 .

Proposition 2.10. The map $\Phi: \operatorname{ker} g \rightarrow \operatorname{Hom}\left(V^{q}, H^{q}\left(\Lambda\left(V^{\leqslant n}\right)\right)\right)$ is an isomorphism.

Proof. First we prove that $\Phi$ is well-defined. Suppose $[\beta]=\left[\beta^{\prime}\right]$ satisfy the conclusion of Lemma 2.9. Since both maps then restrict to the identity on $\Lambda\left(V^{\leqslant n}\right)$, the homotopy $F:(\Lambda(V, \bar{V}, \widehat{V}), D) \rightarrow(\Lambda(V), \partial)$ between them can be chosen so that

$$
F\left(\widehat{V}^{\leqslant n}\right)=F\left(\bar{V}^{\leqslant n}\right)=0 .
$$

Given $v \in V^{q}$, according to (8) we then have

$$
\begin{aligned}
\theta_{\beta^{\prime}}(v)-\theta_{\beta}(v) & =\beta(v)-\beta^{\prime}(v)=F \circ e^{\theta}(v)-F(v) \\
& =F\left(v^{\prime}\right)+F\left(\sum \frac{1}{n !}(S \circ \partial)^{n}(v)\right) \\
& =F(D(s v))+F\left(\sum_{n \geqslant 1} \frac{1}{n !}(S \circ \partial)^{n}(v)\right) \\
& =\partial(F(s v))+F\left(\sum_{n \geqslant 1} \frac{1}{n !}(S \circ \partial)^{n}(v)\right)=\partial(F(s v)) .
\end{aligned}
$$


Thus $\theta_{\beta^{\prime}}(v)-\theta_{\beta}(v)$ is a coboundary. Notice that the relation (10) implies that $F\left(\sum_{n \geqslant 1} \frac{1}{n !}(S \circ \partial)^{n}(v)\right)=0$.

For the injectivity, assume that $\Phi([\beta])(v)=\Phi\left(\left[\beta^{\prime}\right]\right)(v)$ in $H^{q+1}\left(\Lambda\left(V^{\leqslant n}\right)\right)$, then $\theta_{\beta^{\prime}}(v)-\theta_{\beta}(v)=\beta(v)-\beta^{\prime}(v)$ is a coboundary and Lemma 2.5 implies that $[\beta]=\left[\beta^{\prime}\right]$. For the surjectivity, given a homomorphism $\chi \in \operatorname{Hom}\left(V^{q}, H^{q}\left(\Lambda\left(V^{\leqslant n}\right)\right)\right)$, write $\chi(v)=$ $\{\widetilde{\chi(v)}\}$, where $\widetilde{\chi(v)}$ is a cocycle. We define $\beta:(\Lambda(V), \partial) \rightarrow(\Lambda(V), \partial)$ by

$$
\beta(v)=v+\widetilde{\chi(v)} \text { for } v \in V^{q} \quad \text { and } \quad \beta=i d \quad \text { on } \quad V^{\leqslant n} \text {. }
$$

Then $\beta$ is a cochain morphism with $\Phi([\beta])=\chi$.

Finally, given $\beta, \beta^{\prime} \in$ ker g as in Lemma 2.9. So $\beta(v)=v+\theta_{\beta}(v)$ and $\beta^{\prime}(v)=v+$ $\theta_{\beta^{\prime}}(v)$ for $v \in V^{q}$. Therefore, by (9) we get

$$
\beta^{\prime} \circ \beta(v)=v+\theta_{\beta^{\prime}}(v)+\theta_{\beta}(v)=v+\theta_{\beta^{\prime} \circ \beta}(v) .
$$

Consequently, $\Phi\left(\left[\beta^{\prime}\right] .[\beta]\right)=\Phi\left(\left[\beta^{\prime} \circ \beta\right]\right)=\theta_{\beta^{\prime} \circ \beta}=\theta_{\beta^{\prime}}+\theta_{\beta}=\Phi\left(\left[\beta^{\prime}\right]\right)+\Phi([\beta])$. Thus $\Phi$ is a homomorphism of groups.

Summarizing, we have proven:

Theorem 2.11. Let $q>n$ and let $\left(\Lambda\left(V^{q} \oplus V^{\leqslant n}\right), \partial\right)$ be a 1-connected fcca. Then there exists a short exact sequence of groups

$$
\operatorname{Hom}\left(V^{q}, H^{q}\left(\Lambda\left(V^{\leqslant n}\right)\right)\right) \longmapsto \mathcal{E}\left(\Lambda\left(V^{q} \oplus V^{\leqslant n}\right)\right) \stackrel{\mathrm{g}}{\rightarrow} \mathcal{D}_{n}^{q} .
$$

We now focus on the subgroup $\mathcal{E}_{\sharp}\left(\Lambda\left(V^{q} \oplus V^{\leqslant n}\right)\right)$ of $\mathcal{E}\left(\Lambda\left(V^{q} \oplus V^{\leqslant n}\right)\right)$ of the elements inducing the identity on the graded vector space of indecomposables. Let us define $\mathcal{G}_{n}^{q}$ as the subgroup of $\mathcal{E}_{\sharp}\left(\Lambda\left(V^{\leqslant n}\right)\right)$ of those elements $[\alpha]$ satisfying $H^{q+1}(\alpha) \circ$ $b^{q}=b^{q}$ where $b^{q}: V^{q} \rightarrow H^{q+1}\left(\Lambda\left(V^{\leqslant n}\right)\right)$ is as in (2).

Theorem 2.12. Let $q>n$ and let $\left(\Lambda\left(V^{q} \oplus V^{\leqslant n}\right), \partial\right)$ be a 1-connected fcca. Then there exists a short exact sequence of groups

$$
\operatorname{Hom}\left(V^{q}, H^{q}\left(\Lambda\left(V^{\leqslant n}\right)\right)\right) \longmapsto \mathcal{E}_{\sharp}\left(\Lambda\left(V^{q} \oplus V^{\leqslant n}\right)\right) \rightarrow \mathcal{G}_{n}^{q} .
$$

Proof. First let $[\alpha] \in$ kerg. From the relation (7) we deduce that $\widetilde{\alpha}^{q}=i d_{V^{q}}$ and $\alpha^{\leqslant n} \simeq i d_{\Lambda(V \leqslant n)}$, therefore $\alpha^{\leqslant n}$ induces the identity on the indecomposables. So $\widetilde{\alpha}=$ $i d_{V}$. It follows that ker $\mathrm{g} \subseteq \mathcal{E}_{\sharp}\left(\Lambda\left(V^{q} \oplus V^{\leqslant n}\right)\right)$.

Next from (11) we obtain the short exact sequence

$$
\operatorname{Hom}\left(V^{q}, H^{q}\left(\Lambda\left(V^{\leqslant n}\right)\right)\right) \longmapsto \mathcal{E}_{\sharp}\left(\Lambda\left(V^{q} \oplus V^{\leqslant n}\right)\right) \rightarrow \mathrm{g}\left(\mathcal{E}_{\sharp}\left(\Lambda\left(V^{q} \oplus V^{\leqslant n}\right)\right)\right),
$$

where

$$
\mathrm{g}\left(\mathcal{E}_{\sharp}\left(\Lambda\left(V^{q} \oplus V^{\leqslant n}\right)\right)\right)=\left\{\Psi([\alpha])=\left(\widetilde{\alpha}^{q},\left[\alpha^{\leqslant n}\right]\right) \quad \mid[\alpha] \in \mathcal{E}_{\sharp}\left(\Lambda\left(V^{q} \oplus V^{\leqslant n}\right)\right)\right\} .
$$

As $[\alpha] \in \mathcal{E}_{\sharp}\left(\Lambda\left(V^{q} \oplus V^{\leqslant n}\right)\right)$, the graded automorphism $\widetilde{\alpha} \in \operatorname{aut}\left(V^{q} \oplus V^{\leqslant n}\right)$ is the identity which, in turn, implies

$$
\mathrm{g}\left(\mathcal{E}_{\sharp}\left(\Lambda\left(V^{q} \oplus V^{\leqslant n}\right)\right)\right)=\left\{\left(\operatorname{id}_{V^{q}},\left[\alpha^{\leqslant n}\right]\right) \mid\left[\alpha^{\leqslant n}\right] \in \mathcal{E}_{\sharp}\left(\Lambda\left(V^{\leqslant n}\right)\right)\right\} .
$$

As $[\alpha] \in \mathcal{E}_{\sharp}\left(\Lambda\left(V^{q} \oplus V^{\leqslant n}\right)\right)$, the pair $\left(\operatorname{id}_{V^{q}},\left[\alpha^{\leqslant n}\right]\right)$ makes the diagram (3) commute. As a result we can identify $\mathrm{g}\left(\mathcal{E}_{\sharp}\left(\Lambda\left(V^{q} \oplus V^{\leqslant n}\right)\right)\right)$ with the subgroup $\mathcal{G}_{n}^{q}$. 
Corollary 2.13. Let $q>n$ and let $\left(\Lambda\left(V^{q} \oplus V^{\leqslant n}\right), \partial\right)$ be a 1-connected fcca. If $\mathcal{E}_{\sharp}\left(\Lambda\left(V^{\leqslant n}\right)\right)$ is trivial, then

$$
\operatorname{Hom}\left(V^{q}, H^{q}\left(\Lambda\left(V^{\leqslant n}\right)\right)\right) \cong \mathcal{E}_{\sharp}\left(\Lambda\left(V^{q} \oplus V^{\leqslant n}\right)\right) .
$$

Corollary 2.14. Let $q>2 n+1$ and let $\left(\Lambda\left(V^{q} \oplus V^{\leqslant 2 n+1}\right)\right.$, $\left.\partial\right)$ be an $n$-connected $f c c a$. Then

$$
\operatorname{Hom}\left(V^{q}, H^{q}\left(\Lambda\left(V^{\leqslant 2 n+1}\right)\right)\right) \cong \mathcal{E}_{\sharp}\left(\Lambda\left(V^{q} \oplus V^{\leqslant 2 n+1}\right)\right) .
$$

Proof. As $\left(\Lambda\left(V^{q} \oplus V^{\leqslant 2 n+1}\right), \partial\right)$ is $n$-connected, then $V^{1}=\cdots=V^{n}=0$. So, for degree reasons, the group $E_{\sharp}\left(\Lambda\left(V^{\leqslant 2 n+1}\right)\right)$ is trivial and we then apply Corollary 2.13.

\section{Topological applications}

All the $\mathrm{CW}$-complexes which we consider in this section are simply connected having rational homology of finite type.

Let $(\Lambda(V), \partial)$ be a 1-connected fcca. Recall that in [5] it is shown that with $(\Lambda(V), \partial)$ we can associate the following long exact sequence

$$
\cdots \rightarrow V^{n} \stackrel{b^{n}}{\longrightarrow} H^{n+1}\left(\Lambda\left(V^{\leqslant n-1}\right)\right) \rightarrow H^{n+1}(\Lambda(V)) \rightarrow V^{n+1} \stackrel{b^{n+1}}{\longrightarrow} \cdots,
$$

called the Whitehead exact sequence of $(\Lambda(V), \partial)$. Recall that $b^{*}$ is the graded linear map defined in (2).

Now let $X$ be a simply connected rational CW-complex of finite type and let $X^{[n]}$ be the $n$th Postnikov section of $X$. For $q>n$, as in (1), let

$$
X^{[n]} \cup_{\alpha}\left(\bigcup_{i \in I} e_{i}^{q}\right), \quad \text { where } I \text { is finite and } q>n,
$$

be the space obtained by attaching rational cells to $X^{[n]}$ by a map $\alpha: \underset{i \in I}{\vee} S_{\mathbb{Q}}^{q} \rightarrow X^{[n]}$ where $S_{\mathbb{Q}}^{q}$ is the rational sphere of dimension $q$. If $(\Lambda(V), \partial)$ is the Sullivan model of $X$, then it is well-known that $\left(\Lambda\left(V^{\leqslant n}\right), \partial\right)$ is the Sullivan model of $X^{[n]}$ while $\left(\Lambda\left(V^{q} \oplus\right.\right.$ $\left.\left.V^{\leqslant n}\right), \partial\right)$ is the Sullivan model of the pullback $Z^{q}$ of the map $X^{[n]} \rightarrow K\left(\pi_{q}(X) ; q+1\right)$, whose homotopy class is the cohomology class in $H^{q+1}\left(X^{[n]} ; \pi_{q}(X)\right)=\left[X^{[q]} ; K\left(\pi_{q}(X)\right.\right.$; $q+1)$ ] given algebraically by

$$
b^{q} \in \operatorname{Hom}\left(V^{q} ; H^{q+1}\left(\Lambda\left(V^{\leqslant n}\right)\right)\right) \cong H^{q+1}\left(X^{[n]} ; \pi_{q}(X)\right),
$$

over the path fibration:

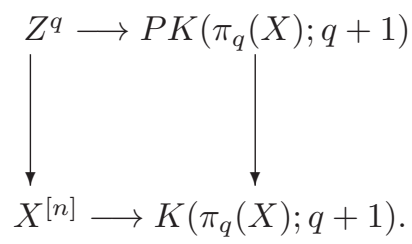

Observe that the fibration long exact sequence implies that $\pi_{q}(X) \cong \pi_{q}\left(Z^{q}\right)$. Hence by the virtues of the properties of the Sullivan model, the Whitehead exact sequence of $\left(\Lambda\left(V^{q} \oplus V^{\leqslant n}\right), \partial\right)$ yields the following exact sequence 


$$
\cdots \stackrel{h^{q}}{\rightarrow} \operatorname{Hom}\left(\pi_{q}(X), \mathbb{Q}\right) \stackrel{b^{q}}{\rightarrow} H^{q+1}\left(X^{[n]}\right) \rightarrow H^{q+1}\left(Z^{q}\right) \stackrel{h^{q+1}}{\rightarrow} \operatorname{Hom}\left(\pi_{q+1}(X), \mathbb{Q}\right) \stackrel{b^{q+1}}{\rightarrow} \cdots,
$$

where $h^{*}$ is the dual of the Hurewicz homomorphism.

Let $D_{n}^{q}$ be the subgroup of $\operatorname{aut}\left(\operatorname{Hom}\left(\pi_{q}(X), \mathbb{Q}\right) \times \mathcal{E}\left(X^{[n]}\right)\right)$ of those pairs $(\xi,[f])$ making the following diagram commute:

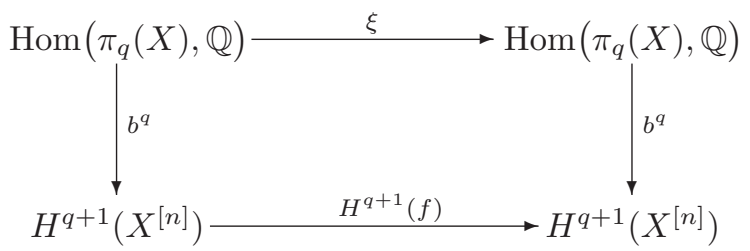

and let

$$
G_{n}^{q}=\left\{[f] \in \mathcal{E}_{\sharp}\left(X^{[n]}\right) \quad \text { such that } \quad H^{q+1}(f) \circ b^{q}=b^{q}\right\} .
$$

Clearly $G_{n}^{q}$ is a subgroup of $\mathcal{E}_{\sharp}\left(X^{[n]}\right)$. From Theorems 2.11 and 2.12 we deduce the following topological results:

Theorem 3.1. Let $X$ be a $C W$-complex. Then for every $n$ and for every $q>n$ there exist two short exact sequence of groups:

$$
\begin{aligned}
\operatorname{Hom}\left(\pi_{q}(X) ; H^{q}\left(X^{[n]}\right)\right) & \longmapsto \mathcal{E}\left(Z^{q}\right) \rightarrow D_{n}^{q}, \\
\operatorname{Hom}\left(\pi_{q}(X) ; H^{q}\left(X^{[n]}\right)\right) & \longmapsto \mathcal{E}_{\sharp}\left(Z^{q}\right) \rightarrow G_{n}^{q} .
\end{aligned}
$$

Moreover, if $\mathcal{E}_{\sharp}\left(X^{[n]}\right)$ is a trivial group, then $\operatorname{Hom}\left(\pi_{q}(X) ; H^{q}\left(X^{[n]}\right)\right) \cong \mathcal{E}_{\sharp}\left(Z^{q}\right)$. Here $Z^{q}$ is the space given in (12).

Proof. The two sequences (16) follow from Theorems 2.11 and 2.12. Notice that Sullivan theory implies the following identifications:

$$
\begin{gathered}
\mathcal{E}\left(Z^{q}\right) \cong \mathcal{E}\left(\Lambda\left(V^{q} \oplus V^{\leqslant n}\right)\right), \quad E_{\sharp}\left(Z^{q}\right) \cong \mathcal{E}_{\sharp}\left(\Lambda\left(V^{q} \oplus V^{\leqslant n}\right)\right), \\
D_{n}^{q} \cong \mathcal{D}_{n}^{q}, \quad G_{n}^{q} \cong \mathcal{G}_{n}^{q} .
\end{gathered}
$$

Finally, the last assertion follows by applying Corollary 2.13.

Remark 3.2. According to Theorem 3.1, if we take $X^{[n]}=K(\pi, n)$, where $\pi$ is a vector space of finite dimension, then we get

$$
\mathcal{E}_{\sharp}\left(Z^{q}\right) \cong \operatorname{Hom}\left(\pi_{q}(X) ; H^{q}(K(\pi, n))\right) .
$$

Indeed, we know that the Sullivan model of $K(\pi, n)$ is $\left(\Lambda\left(V^{n}\right), 0\right)$ where $V^{n} \cong$ $H^{n}(K(\pi, n), \mathbb{Q})$. Therefore the group $\mathcal{E}_{\sharp}(K(\pi, n)) \cong \mathcal{E}_{\sharp}\left(\Lambda\left(V^{n}\right)\right)$ is trivial.

Let $X$ be a simply connected rational CW-complex of finite type. As the space $Z^{n+1}$ coincides with $X^{[n+1]}$, Theorem 3.1 implies:

Corollary 3.3. Let $X$ be a simply connected rational $C W$-complex of finite type. The following two short sequences are exact:

$$
\begin{aligned}
\operatorname{Hom}\left(\pi_{q}(X) ; H^{q}\left(X^{[n]}\right)\right) & \longmapsto \mathcal{E}\left(X^{[n+1]}\right) \rightarrow D_{n-1}^{n}, \\
\operatorname{Hom}\left(\pi_{q}(X) ; H^{q}\left(X^{[n]}\right)\right) & \longmapsto \mathcal{E}_{\sharp}\left(X^{[n+1]}\right) \rightarrow \mathrm{G}_{n-1}^{n} .
\end{aligned}
$$

Moreover, if $\mathcal{E}_{\sharp}\left(X^{[n-1]}\right)$ is finite, then $\operatorname{Hom}\left(\pi_{q}(X) ; H^{q}\left(X^{[n]}\right)\right) \cong \mathcal{E}_{\sharp}\left(X^{[n]}\right)$. 
Now let us consider the dual of the Hurewicz homomorphism $h^{*}$ given in the long exact sequence $(13)$ and let $\mathcal{E}_{\sharp}^{(q+1)}\left(X^{[n]}\right)$ denote the subgroup of $\mathcal{E}_{\sharp}\left(X^{[n]}\right)$ consisting of the self-homotopy equivalences $[f]$ such that $H^{q+1}(f): H^{q+1}\left(X^{[n]}\right) \rightarrow H^{q+1}\left(X^{[n]}\right)$ is the identity.

Corollary 3.4. Let $X$ be a simply connected rational $C W$-complex and let $Z^{q}$ be the space given in (12). Assume that $h^{q}$ is nil and $h^{q+1}$ is injective. There exist two short exact sequence of groups:

$$
\begin{aligned}
\operatorname{Hom}\left(\pi_{q}(X) ; H^{q}\left(X^{[n]}\right)\right) & \longmapsto \mathcal{E}\left(Z^{q}\right) \rightarrow \mathcal{E}^{(q+1)}\left(X^{[n]}\right), \\
\operatorname{Hom}\left(\pi_{q}(X) ; H^{q}\left(X^{[n]}\right)\right) & \longmapsto \mathcal{E}_{\sharp}\left(Z^{q}\right) \rightarrow \mathcal{E}_{\sharp}^{(q+1)}\left(X^{[n]}\right) .
\end{aligned}
$$

Proof. First notice that if $h^{q}$ is nil and $h^{q+1}$ is injective, then according to the long exact sequence $(13)$ the map $b^{q}: \operatorname{Hom}\left(\pi_{q}(X), \mathbb{Q}\right) \rightarrow H^{q+1}\left(X^{[n]}\right)$ is an isomorphism. Then for every $[f] \in \mathcal{E}\left(X^{n}\right)$ the pair $\left(\left(b^{q}\right)^{-1} \circ H^{q+1}(f) \circ b^{q},[f]\right)$ makes the diagram (14) commute. Therefore we get a map $\mathcal{E}\left(X^{n}\right) \rightarrow D_{n}^{q}$ defined by $[f] \mapsto$ $\left(\left(b^{q}\right)^{-1} \circ H^{q+1}(f) \circ b^{q},[f]\right)$ and it is easy to see that it is an isomorphism of groups.

Likewise by (15) we can say that the group $G_{n}^{q}$ coincides with the subgroup $\mathcal{E}_{\sharp}^{(q+1)}\left(X^{n}\right)$. Thus the sequences (16) imply the sequences (17).

Corollary 3.5. Let $X$ be simply connected rational $C W$-complex of finite order. Assume that $\pi_{i}(X) \neq 0$ for $i=n-1, n, n+1$. If $\mathcal{E}\left(X^{[n]}\right)$ is finite, then $\mathcal{E}\left(X^{[n+1]}\right)$ and $\mathcal{E}\left(X^{[n-1]}\right)$ are infinite.

Proof. Working algebraically, we assume given a fcca $(\Lambda(V), \partial)$ with $V^{i} \neq 0$ for $i=n-1, n, n+1$ and suppose that $\mathcal{E}\left(\Lambda\left(V^{\leqslant n+1}\right)\right)$ is finite. We prove $\mathcal{E}\left(\Lambda\left(V^{\leqslant n}\right)\right)$ is infinite. The result then follows from the properties of the Sullivan model.

Since $\mathcal{E}\left(\Lambda\left(V^{\leqslant n+1}\right)\right)$ is finite, applying Theorem 2.11 gives that $H^{n+1}\left(\Lambda\left(V^{\leqslant n-1}\right)\right)=$ 0 . This implies the the linear map $b^{n}: V^{n} \rightarrow H^{n+1}\left(\Lambda\left(V^{\leqslant n-1}\right)\right)=0$ vanishes. Now by taking $\alpha=\mathrm{id}:\left(\Lambda\left(V^{\leqslant n-1}\right), \partial\right) \rightarrow\left(\Lambda\left(V^{\leqslant n-1}\right), \partial\right)$ and $\xi^{a} \in \operatorname{aut}\left(V^{n}\right), a \in \mathbb{Q}$, such that $\xi^{a}(v)=a v$ for $v \in V^{n}$, the following diagram commutes obviously:

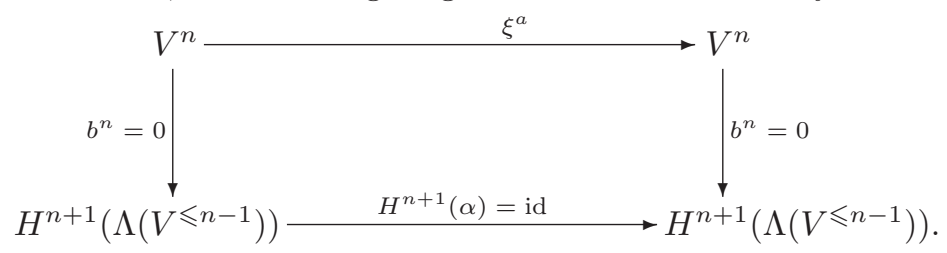

Therefore there exist an infinity of pairs $\left(\xi^{a}\right.$, [id] $) \in \mathcal{D}_{n-1}^{n}$, so the group $\mathcal{D}_{n-1}^{n}$ is infinite, it follows that $\mathcal{E}\left(\Lambda\left(V^{\leqslant n}\right)\right)$ is infinite by Theorem 2.11. Finally, if $\mathcal{E}\left(\Lambda\left(V^{\leqslant n-1}\right)\right)$ is finite, then by the above argument the group $\mathcal{E}\left(\Lambda\left(V^{\leqslant n}\right)\right)$ must be infinite. Contradiction.

Corollary 3.6. Let $X$ be a simply connected rational $C W$ complex of finite type. Assume that $\mathcal{E}\left(Z^{n+2}\right)$ is finite, then the space $X^{[n+1]}$ has the homotopy type of $X^{[n]} \times$ $K\left(\pi_{n+1}(X), n+1\right)$.

Proof. As $\mathcal{E}\left(Z^{n+2}\right)$ is finite, Theorem 3.1 implies that

$$
\operatorname{Hom}\left(\pi_{q}(X) ; H^{q}\left(X^{[n]}\right)\right)=H^{n+2}\left(X^{[n]}, \pi_{n+1}(X)\right)=0 .
$$


This implies that the Postnikov invariant $\left[k^{n}\right] \in H^{n+2}\left(X^{[n]}, \pi_{n+1}(X)\right)$ is nil. As a result the space $X^{[n+1]}$ has the homotopy type of $X^{[n]} \times K\left(\pi_{n+1}(X), n+2\right)$.

Remark 3.7. Let $X$ be as in Corollary 3.6. Then if the Postnikov invariant $\left[k^{n+1}\right]$ is not nil, then $\mathcal{E}\left(Z^{n+2}\right)$ is infinite.

\subsection{Realization problem}

Recall that the realizability problem for groups deals with the following question: Given a group $G$, is there a space $X$ such that $G=\mathcal{E}(X)$ ? A complete answer to the realizability problem for finite groups is given by Costoya-Viruel [9]. Here, in this section, we give an alternative proof based on the main result of this work.

Let $G=\left\{g_{1}, g_{2}, \ldots, g_{n}\right\}$ be a group of order $n$. By Cayley's theorem there is a monomorphism $G \rightarrow S_{n}$ given by $g_{s} \mapsto \sigma_{s}: g_{k} \longrightarrow g_{s} g_{k}, 1 \leqslant k \leqslant n$. For $2 \leqslant s \leqslant n$ write $\sigma_{s}=\left(\begin{array}{cccc}1 & 2 & \cdots & n \\ s & \sigma_{s}(2) & \cdots & \sigma_{s}(n)\end{array}\right)$ and let

$$
\sigma_{2}=\left(12 \sigma_{1}(2) \ldots, \sigma_{2}^{\kappa_{2}}(2)\right)\left(i_{1} \sigma_{1}\left(i_{1}\right) \ldots \sigma_{2}^{\kappa_{i_{1}}}\left(i_{1}\right)\right) \ldots\left(i_{k} \sigma_{1}\left(i_{k}\right) \ldots \sigma_{2}^{\kappa_{i_{k}}}\left(i_{k}\right)\right)
$$

be the decomposition of $\sigma_{2}$ as a product of cycles. Notice that the monomorphism $G \rightarrow S_{n}$ implies that

$$
\sigma_{\sigma_{s}(i)}(j)=\sigma_{s} \circ \sigma_{i}(j), \quad 1 \leqslant i, j, s \leqslant n .
$$

For a group $G$, we define $\left(\Lambda\left(x_{1}, x_{2}, y_{1}, y_{2}, y_{3},\left\{z_{j}, w_{j}\right\}_{1 \leqslant j \leqslant n}\right), \partial\right)$, where $\left|x_{1}\right|=8,\left|x_{2}\right|=$ $10,\left|w_{j}\right|=40$, by

$$
\begin{aligned}
& \partial\left(x_{1}\right)=\partial\left(x_{2}\right)=\partial\left(w_{j}\right)=0, \quad \partial\left(y_{1}\right)=x_{1}^{3} x_{2}, \quad \partial\left(y_{2}\right)=x_{1}^{2} x_{2}^{2}, \quad \partial\left(y_{3}\right)=x_{1} x_{2}^{3}, \\
& \partial\left(z_{1}\right)=w_{1}^{3}+w_{1} w_{2} x_{2}^{4}+\sum_{\tau=1}^{k} w_{1} w_{i_{\tau}} x_{2}^{4}+u+x_{1}^{15}, \quad u=y_{1} y_{2} x_{1}^{4} x_{2}^{2}-y_{1} y_{3} x_{1}^{5} x_{2}+y_{2} y_{3} x_{1}^{6}, \\
& \partial\left(z_{j}\right)=w_{j}^{3}+w_{j} w_{\sigma_{j}(2)} x_{2}^{4}+\sum_{\tau=1}^{k} w_{j} w_{\sigma_{s}\left(i_{\tau}\right)} x_{2}^{4}+u+x_{1}^{15}, \quad 2 \leqslant j \leqslant n .
\end{aligned}
$$

Thus applying Theorem 2.11 to the fcca $\left(\Lambda\left(x_{1}, x_{2}, y_{1}, y_{2}, y_{3},\left\{z_{j}, w_{j}\right\}_{1 \leqslant j \leqslant n}\right), \partial\right)$ we get the following exact sequence

$$
\begin{aligned}
H^{119}\left(\Lambda\left(x_{1}, x_{2}, y_{1}, y_{2}, y_{3}, w_{1}, \ldots, w_{n}\right)\right) & \longmapsto \mathcal{E}\left(\Lambda\left(x_{1}, x_{2}, y_{1}, y_{2}, y_{3},\left\{z_{j}, w_{j}\right\}_{1 \leqslant j \leqslant n}\right)\right) \\
& \rightarrow \mathcal{D}_{40}^{119} .
\end{aligned}
$$

Lemma 3.8. $H^{119}\left(\Lambda\left(x_{1}, x_{2}, y_{1}, y_{2}, y_{3}, w_{1}, \ldots, w_{n}\right)\right)=0$.

Proof. First $\Lambda^{119}$ is spanned by

$y_{1} x_{1}^{7} x_{2}^{3}, y_{1} x_{1}^{2} x_{2}^{7}, y_{2} x_{1}^{3} x_{2}^{6}, y_{2} x_{1}^{8} x_{2}^{2}, y_{3} x_{1}^{9} x_{2}, y_{3} x_{1}^{4} x_{2}^{5}, y_{1} w_{j} x_{1}^{2} x_{2}^{3}, y_{2} w_{j} x_{1}^{3} x_{2}^{2}, y_{3} w_{j} x_{1}^{4} x_{2}$.

Next, if

$$
\begin{aligned}
\phi= & A_{1} y_{1} x_{1}^{7} x_{2}^{3}+A_{2} y_{1} x_{1}^{2} x_{2}^{7}+A_{3} y_{2} x_{1}^{3} x_{2}^{6}+A_{4} y_{2} x_{1}^{8} x_{2}^{2}+A_{5} y_{3} x_{1}^{9} x_{2}+A_{6} y_{3} x_{1}^{4} x_{2}^{5} \\
& +\left(\sum_{j=1}^{n} A_{7}^{(j)} y_{1} w_{j} x_{1}^{2} x_{2}^{3}\right)+\left(\sum_{j=1}^{n} A_{8}^{(j)} y_{2} w_{j} x_{1}^{3} x_{2}^{2}\right)+\left(\sum_{j=1}^{n} A_{9}^{(j)} y_{3} w_{j} x_{1}^{4} x_{2}\right),
\end{aligned}
$$


it follows that

$$
\begin{aligned}
\partial(\phi)=\left(A_{1}+A_{4}+A_{5}\right) x_{1}^{10} x_{2}^{4}+ & \left(A_{2}+A_{3}+A_{6}\right) x_{1}^{5} x_{2}^{8} \\
+ & \left(\sum_{j=1}^{n} A_{7}^{(j)}+A_{8}^{(j)}+A_{9}^{(j)}\right) w_{j} x_{1}^{5} x_{2}^{4} .
\end{aligned}
$$

So the space of 119 -cocycles is spanned by $y_{3} x_{1}^{9} x_{2}-y_{1} x_{1}^{7} x_{2}^{3}, y_{2} x_{1}^{2} x_{2}^{8}-y_{1} x_{1}^{7} x_{2}^{3}$, $y_{1} x_{1}^{2} x_{2}^{7}-y_{3} x_{1}^{4} x_{2}^{5}, y_{2} x_{1}^{3} x_{2}^{6}-y_{3} x_{1}^{4} x_{2}^{5}, y_{3} w_{j} x_{1}^{4} x_{2}-y_{1} w_{j} x_{1}^{2} x_{2}^{3}, y_{2} w_{j} x_{1}^{3} x_{2}^{2}-y_{1} w_{j} x_{1}^{2} x_{2}^{3}$.

But we have

$$
\begin{aligned}
\partial\left(y_{3} y_{1} x_{2}^{6}\right) & =y_{1} x_{1}^{7} x_{2}^{3}-y_{3} x_{1}^{9} x_{2}, & & \partial\left(y_{2} y_{1} x_{1}^{5} x_{2}\right)=y_{2} x_{1}^{8} x_{2}^{2}-y_{1} x_{1}^{7} x_{2}^{3}, \\
\partial\left(y_{3} y_{1} x_{1} x_{2}^{4}\right) & =y_{1} x_{1}^{2} x_{2}^{7}-y_{3} x_{1}^{4} x_{2}^{5}, & & \partial\left(y_{2} y_{3} x_{1}^{2} x_{2}^{3}\right)=y_{2} x_{1}^{3} x_{2}^{6}-y_{3} x_{1}^{4} x_{2}^{5}, \\
\partial\left(y_{1} y_{2} w_{j} x_{2}\right) & =y_{2} w_{j} x_{1}^{3} x_{2}^{2}-y_{1} w_{j} x_{1}^{2} x_{2}^{3}, & \partial\left(y_{1} y_{3} w_{j} x_{1}\right) & =y_{3} w_{j} x_{1}^{4} x_{2}-y_{1} w_{j} x_{1}^{2} x_{2}^{3}
\end{aligned}
$$

and the lemma is proved.

Let $g_{s} \in G$. For every $j \leqslant n$, define

$$
\xi_{s}\left(z_{j}\right)=z_{\sigma_{s}(j)}, \quad \alpha_{s}\left(w_{j}\right)=w_{\sigma_{s}(j)}, \quad \alpha_{s}=i d \quad \text { on } \quad x_{1}, x_{2}, y_{1}, y_{2}, y_{3} .
$$

Clearly, $\left[\alpha_{s}\right] \in \mathcal{E}\left(\Lambda\left(x_{1}, x_{2}, y_{1}, y_{2}, y_{3}, w_{1}, \ldots, w_{n}\right)\right)$ and $b^{119} \circ \xi_{s}=H^{120}\left(\alpha_{s}\right) \circ b^{119}$. So we get a map $\Omega: G \longrightarrow \mathcal{D}_{40}^{119}$ defined by $\Omega\left(g_{s}\right)=\left(\xi_{s},\left[\alpha_{s}\right]\right)$.

Proposition 3.9. $\Omega$ is an isomorphism of groups.

Proof. Let $(\xi,[\alpha]) \in \mathcal{D}_{40}^{119}$. For degree reasons we have

$\alpha\left(x_{1}\right)=\beta_{1} x_{1}, \quad \alpha\left(x_{2}\right)=\beta_{2} x_{2}, \quad \alpha\left(y_{1}\right)=p_{1} y_{1}, \quad \alpha\left(y_{2}\right)=p_{2} y_{2}, \quad \alpha\left(y_{3}\right)=p_{3} y_{3}$,

$\alpha\left(w_{j}\right)=a_{j 1} w_{1}+\cdots+a_{j n} w_{n}+\gamma_{j 1} x_{1}^{5}+\gamma_{j 2} x_{2}^{4}, \quad \xi\left(z_{j}\right)=\lambda_{j 1} z_{1}+\cdots+\lambda_{j n} z_{n}$.

As $\alpha\left(\partial\left(y_{i}\right)\right)=\partial\left(\alpha\left(y_{i}\right)\right)$ it follows that

$$
p_{1}=\beta_{1}^{3} \beta_{2}, \quad p_{2}=\beta_{1}^{2} \beta_{2}^{2}, \quad p_{3}=c_{1}=\beta_{1} \beta_{2}^{3} .
$$

Thus

$$
\begin{aligned}
& \alpha\left(\partial\left(z_{j}\right)\right)=\alpha\left(w_{j}^{3}\right)+ \alpha\left(w_{j} w_{\sigma_{j}(2)} x_{2}^{4}\right)+\alpha\left(\sum_{\tau=1}^{k} w_{j} w_{\sigma_{j}\left(i_{\tau}\right)} x_{2}^{4}\right)+\alpha(u)+\alpha\left(x_{1}^{15}\right), \\
& \partial\left(\xi\left(z_{j}\right)\right)=\lambda_{j 1}\left(w_{1}^{3}+w_{1} w_{2} x_{2}^{4}+\sum_{\tau=1}^{k} w_{1} w_{\sigma_{s}\left(i_{\tau}\right)} x_{2}^{4}+u+x_{1}^{15}\right)+\cdots \\
&+\lambda_{j n}\left(w_{n}^{3}+w_{n} w_{\sigma_{n}(2)} x_{2}^{4}+\sum_{\tau=1}^{k} w_{n} w_{\sigma_{s}\left(i_{\tau}\right)} x_{2}^{4}+v^{2}+u+x_{1}^{15}\right) .
\end{aligned}
$$

As $(\xi,[\alpha]) \in \mathcal{D}_{40}^{119}$ and due to (5) there exists $\varphi_{j} \in \Lambda^{119}\left(x_{1}, x_{2}, y_{1}, y_{2}, y_{3}, w_{1}, \ldots, w_{n}\right)$ such that

$$
\partial\left(\xi\left(z_{j}\right)\right)-\alpha\left(\partial\left(z_{j}\right)\right)=\partial\left(\varphi_{j}\right), \quad \forall j \leqslant n .
$$

By expanding $\alpha\left(w_{j}^{3}\right)=\left(a_{j 1} w_{1}+\cdots+a_{j n} w_{n}+\gamma_{j 1} x_{1}^{5}+\gamma_{j 2} x_{2}^{4}\right)^{3}$ the monomials $a_{j i}^{2} a_{j s} w_{s}^{2} w_{t}, \gamma_{j 1}^{2} a_{j s} x_{1}^{10} w_{s}, \gamma_{j 2}^{3} x_{2}^{12}$ where $1 \leqslant s \neq t \leqslant n$ appear. As $\alpha$ is a homotopy 
equivalence it induces an isomorphism on the indecomposables. So $a_{j 1}, \ldots, a_{j n}$ cannot be all nil. Equating the coefficients in (24) and (21) leads to

$$
a_{j s}^{2} a_{j t}=0, \quad \gamma_{j 1}=\gamma_{j 2}=0, \quad 1 \leqslant s \neq t \leqslant n, \quad 1 \leqslant j \leqslant n .
$$

As a result if $a_{1 s} \neq 0$, then $a_{1 j}=0$ for every $1 \leqslant s \neq j \leqslant n$. It follows that $\alpha\left(w_{1}\right)=$ $a_{1 s} w_{s}$ and

$$
\begin{aligned}
& \alpha\left(\partial\left(z_{1}\right)\right)=a_{1 s}^{3} w_{s}^{3}+a_{1 s} w_{s} \alpha\left(w_{2} x_{2}^{4}\right)+\sum_{\tau=1}^{k} a_{1 s} w_{s} \alpha\left(w_{i_{\tau}} x_{2}^{4}\right)+\alpha(u)+\alpha\left(x_{1}^{15}\right), \\
& \partial\left(\xi\left(z_{1}\right)\right)=\lambda_{s 1}\left(w_{s}^{2}+w_{s} w_{\sigma_{s}(2)} x_{2}^{4}+\sum_{\tau=1}^{k} w_{s} w_{\sigma_{t}\left(i_{\tau}\right)} x_{2}^{4}+u+x_{1}^{15}\right)+\cdots
\end{aligned}
$$

Notice the relations (23) imply that $\alpha(u)=\beta_{1}^{9} \beta_{2}^{5}(u)$ and $\alpha\left(x_{1}^{15}\right)=\beta_{1}^{15}$. Consequently, equating the coefficients in (26) and using (22), (23), for every $\tau \leqslant k$ and for every $2 \leqslant j \leqslant n$ we get

$$
a_{1 s}^{3}=a_{1 s} a_{2 \sigma_{s}(2)} \beta_{2}^{4}=a_{1 s} a_{i_{\tau} \sigma_{s}\left(i_{\tau}\right)} \beta_{2}^{4}=\beta_{1}^{9} \beta_{2}^{5}=\beta_{1}^{15}=\lambda_{s 1}, \quad \lambda_{s j}=0
$$

it follows that $a_{2 \sigma_{s}(2)} \neq 0$ and $a_{i_{\tau} \sigma_{s}\left(i_{\tau}\right)} \neq 0$ for every $\tau \leqslant k$. So

$$
\alpha\left(w_{2}\right)=a_{2 \sigma_{s}(2)} w_{2}, \quad \alpha\left(w_{i_{\tau}}\right)=a_{i_{\tau} \sigma_{s}\left(i_{\tau}\right)} w_{i_{\tau}} .
$$

Likewise we have

$$
\begin{gathered}
\alpha\left(\partial\left(z_{2}\right)\right)=a_{2 \sigma_{s}(2)}^{3} w_{\sigma_{s}(2)}^{3}+a_{2 \sigma_{s}(2)} w_{\sigma_{s}(2)} \alpha\left(w_{\sigma_{2}(2)} x_{2}^{4}\right) \\
+\sum_{\tau=1}^{k} a_{2 \sigma_{s}(2)} w_{\sigma_{s}(2)} \alpha\left(w_{\sigma_{2}\left(i_{\tau}\right)} x_{2}^{4}\right)+\alpha(u)+\alpha\left(x_{1}^{15}\right), \\
\partial\left(\xi\left(z_{2}\right)\right)=\lambda_{\sigma_{s}(2) 2}\left(w_{\sigma_{s}(2)}^{3}+w_{\sigma_{s}(2)} w_{\sigma_{\sigma_{s}(2)}(2)} x_{2}^{4}\right. \\
\left.+\sum_{\tau=1}^{k} w_{\sigma_{s}(2)} w_{\sigma_{\sigma_{s}(2)}\left(i_{\tau}\right)} x_{2}^{4}+u+x_{1}^{15}\right)+\ldots
\end{gathered}
$$

Due to (19) we can write:

$$
w_{\sigma_{\sigma_{s}(2)}(2)}=w_{\sigma_{s}\left(\sigma_{2}(2)\right)}, \quad w_{\sigma_{\sigma_{s}(2)}\left(i_{\tau}\right)}=w_{\sigma_{s}\left(\sigma_{2}\left(i_{\tau}\right)\right)}, \quad \tau \leqslant k .
$$

Taking in consideration (25) and comparing the coefficients in (28) we get

$$
\begin{gathered}
a_{2 \sigma_{s}(2)}^{3}=a_{2 \sigma_{s}(2)} a_{\sigma_{s}(2), \sigma_{s}\left(\sigma_{2}(2)\right)} \beta_{2}^{4}=a_{2 \sigma_{s}(2)} a_{\sigma_{s}\left(i_{\tau}\right), \sigma_{s}\left(\sigma_{2}\left(i_{\tau}\right)\right)} \beta_{2}^{4}=\beta_{1}^{9} \beta_{2}^{5}=\beta_{1}^{15}=\lambda_{\sigma_{s}(2) 2}, \\
\lambda_{\sigma_{s}(2) j}=0, \quad 1 \leqslant j \neq 2 \leqslant n, \quad \forall \tau \leqslant k .
\end{gathered}
$$

Now set $\sigma_{2}^{p}(2)=r_{p}$. By iterating the above process we get

$$
\begin{aligned}
a_{r_{p} \sigma_{s}\left(r_{p}\right)}^{3} & =a_{r_{p} \sigma_{s}\left(r_{p}\right)} a_{\sigma_{s}\left(r_{p}\right), \sigma_{s}\left(\sigma_{2}\left(r_{p}\right)\right)} \beta_{2}^{4}=a_{r_{p} \sigma_{s}\left(r_{p}\right)} a_{\sigma_{s}\left(\sigma_{2}^{p}\left(i_{\tau}\right)\right), \sigma_{s}\left(\sigma_{2}^{p+1}\left(i_{\tau}\right)\right)} \beta_{2}^{4} \\
& =\beta_{1}^{9} \beta_{2}^{5}=\beta_{1}^{15}=\lambda_{\sigma_{s}\left(r_{p}\right) r_{p}}, \quad \lambda_{\sigma_{s}\left(r_{p}\right) j}=0, \quad 1 \leqslant j \neq r_{p} \leqslant n .
\end{aligned}
$$

Now comparing (27), (29) and (30), for all $1 \leqslant \tau \leqslant k$ we get

$$
\begin{aligned}
a_{1 s} & =a_{2 \sigma_{s}(2)}=a_{r_{p} \sigma_{s}\left(r_{p}\right)}=\beta_{2}=\beta_{1}=\lambda_{s 1}=\lambda_{\sigma_{s}(2) 2}=\lambda_{\sigma_{s}\left(r_{p}\right) r_{p}}=1, \\
a_{i_{\tau} \sigma_{s}\left(i_{\tau}\right)} & =a_{\sigma_{s}\left(i_{\tau}\right), \sigma_{s}\left(\sigma_{2}\left(i_{\tau}\right)\right)}=a_{\sigma_{s}\left(\sigma_{2}^{p}\left(i_{\tau}\right)\right), \sigma_{s}\left(\sigma_{2}^{p+1}\left(i_{\tau}\right)\right)}=\lambda_{\sigma_{s}\left(\sigma_{2}^{p+1}\left(i_{\tau}\right)\right), \sigma_{s}\left(\sigma_{2}^{p}\left(i_{\tau}\right)\right)}=1 .
\end{aligned}
$$


Hence, for every $\tau \leqslant k$ and $p \leqslant \kappa_{2}$,

$$
\alpha\left(w_{\sigma_{2}^{p}(2)}\right)=w_{\sigma_{s}\left(\sigma_{2}^{p}\left(i_{2}\right)\right)}, \quad \alpha\left(w_{\sigma_{2}^{p}\left(i_{\tau}\right)}\right)=w_{\sigma_{s}\left(\sigma_{2}^{p}\left(i_{\tau}\right)\right)} .
$$

Finally, due to (18) we can say that every $1 \leqslant j \leqslant n$ occurs as $\sigma_{2}^{p}(2)$ or $\sigma_{2}^{p}\left(i_{\tau}\right)$, where $\tau \leqslant k$, for a certain $p$. Therefore from (31) we deduce that $\alpha\left(w_{j}\right)=\alpha\left(w_{\sigma_{s}(j)}\right)$.

Summarizing, we have proven that if $(\xi,[\alpha]) \in \mathcal{D}_{40}^{119}$, then there exists a permutation $\sigma_{s}=\left(\begin{array}{cccc}1 & 2 & \cdots & n \\ s & \sigma_{s}(2) & \cdots & \sigma_{s}(n)\end{array}\right)$ such that

$$
\xi\left(z_{j}\right)=z_{\sigma_{s}(j)}, \quad \alpha\left(w_{j}\right)=w_{\sigma(j)}, \quad \alpha=i d, \quad \text { on } \quad x_{1}, x_{2}, y_{1}, y_{2}, y_{3} .
$$

This allows us to define $\Omega^{\prime}: \mathcal{D}_{40}^{119} \rightarrow G$ by setting $\Omega^{\prime}(\xi,[\alpha])=g_{s}$, where the element $g_{s}$ corresponds to the permutation $\sigma_{s}$ via Cayley theorem. Clearly $\Omega^{\prime}$ is the inverse of $\Omega$ and as $g_{s} g_{s^{\prime}}$ correspond to the permutation $\sigma_{s} \circ \sigma_{s^{\prime}}$ it is easy to see that $\Omega$ is an isomorphism.

Applying Theorem 2.11, Lemma 3.8 and Proposition 3.9 we deduce that

$$
\mathcal{E}\left(\Lambda\left(x_{1}, x_{2}, y_{1}, y_{2}, y_{3},\left\{z_{j}, w_{j}\right\}_{1 \leqslant j \leqslant n}\right)\right) \cong G
$$

and by the Sullivan model there exists a simply connected CW-complex $X$ such that $\mathcal{E}\left(X_{\mathbb{Q}}\right) \cong G$.

Remark 3.10. The fcca $\left(\Lambda\left(x_{1}, x_{2}, y_{1}, y_{2}, y_{3},\left\{z_{j}, w_{j}\right\}_{1 \leqslant j \leqslant n}\right), \partial\right)$ given in (20), which is not elliptic, is a little modification of the elliptic fcca used by Costoya-Viruel in [9] to show their main result.

Using the arguments of the proof of Proposition 3.9 and omitting the details we can prove that:

Example 3.11. If we define $\left(\Lambda\left(x_{1}, x_{2}, y_{1}, y_{2}, y_{3},\left\{z_{n}, w_{n}\right\}_{n \in \mathbb{Z}}\right), \partial\right)$, where $\left|x_{1}\right|=8,\left|x_{2}\right|=$ $10,\left|w_{n}\right|=40$ for all $n \in \mathbb{Z}$, by

$$
\begin{aligned}
& \partial\left(x_{1}\right)=\partial\left(x_{2}\right)=\partial\left(w_{n}\right)=0, \quad \partial\left(y_{1}\right)=x_{1}^{3} x_{2}, \quad \partial\left(y_{2}\right)=x_{1}^{2} x_{2}^{2}, \quad \partial\left(y_{3}\right)=x_{1} x_{2}^{3}, \\
& \partial\left(z_{n}\right)=w_{n}^{3}+w_{n} w_{n+1} x_{2}^{4}+y_{1} y_{2} x_{1}^{4} x_{2}^{2}-y_{1} y_{3} x_{1}^{5} x_{2}+y_{2} y_{3} x_{1}^{6}+x_{1}^{15},
\end{aligned}
$$

then

$$
\mathcal{E}\left(\Lambda\left(x_{1}, x_{2}, y_{1}, y_{2}, y_{3},\left\{z_{n}, w_{n}\right\}_{n \in \mathbb{Z}}\right)\right) \cong \mathbb{Z}
$$

Indeed, let $m \in \mathbb{Z}$. For every $n \in \mathbb{Z}$, define

$$
\xi_{m}\left(z_{n}\right)=z_{n+m}, \quad \alpha_{m}\left(w_{n}\right)=w_{n+m}, \quad \alpha_{m}=i d \quad \text { on } \quad x_{1}, x_{2}, y_{1}, y_{2}, y_{3},
$$

so that we get a homomorphism:

$$
\Omega: \mathbb{Z} \rightarrow \mathcal{D}_{40}^{119}, \quad \Omega(m)=\left(\xi_{m},\left[\alpha_{m}\right]\right) .
$$

Now if $(\xi,[\alpha]) \in \mathcal{D}_{40}^{119}$, then there is a unique $m \in \mathbb{Z}$ such that

$$
\xi\left(z_{n}\right)=z_{n+m}, \quad \alpha\left(w_{n}\right)=w_{n+m}, \quad \alpha=i d \quad \text { on } \quad x_{1}, x_{2}, y_{1}, y_{2}, y_{3} .
$$

So $\Omega$ is an isomorphism. Finally, (32) follows by applying Theorem 2.11 and Lemma 3.8 . 


\section{References}

[1] D.J. Anick, Hopf algebras up to homotopy, J. Amer. Math. Soc. 2 (1989), 417453.

[2] M. Arkowitz and G. Lupton, Rational obstruction theory and rational homotopy sets, Math. Z. 235(3) (2000), 525-539.

[3] M. Arkowitz and K. Maruyama, Self-homotpy equivalences which induce the identity on the homology, cohomology or homotopy groups, Topology Appl. 87 (1998), 133-154.

[4] M. Arkowitz, Introduction to Homotopy Theory, Springer-Verlag, New York, 2011.

[5] M. Benkhalifa, Cardinality of rational homotopy types of simply connected $C W$ complexes, Internat. J. Math. 22(2) (2011), 179-193.

[6] M. Benkhalifa, Realizability of the group of rational self-homotopy equivalences, J. Homotopy Relat. Struct. 5(1) (2010), 361-372.

[7] M. Benkhalifa, Rational self-homotopy equivalences and Whitehead exact sequence, J. Homotopy Relat. Struct. 4(1) (2009), 135-144.

[8] M. Benkhalifa and S.B. Smith, The effect of cell attachment on the group of self-equivalences of an R-local space. J. Homotopy Relat. Struct. 10(3) (2015), 549-564.

[9] C. Costoya and A. Viruel, Every finite group is the group of self homotopy equivalences of an elliptic space, Acta Math. 213 (2014), 49-62.

[10] Y. Félix, S. Halperin, and J.-C. Thomas, Rational Homotopy Theory, Grad. Texts in Math., Vol. 205, Springer-Verlag, New York, 2001.

[11] J. Rutter, Spaces of Homotopy Self-Equivalences: A Survey, Lecture Notes in Math., Vol. 162, Springer-Verlag, Berlin, 1997.

[12] D. Sullivan, infinitesimal computations in topology. Publ. Math. Inst. Hautes Études Sci. 47 (1977), 117-181.

Mahmoud Benkhalifa mbenkhalifa@sharjah.ac.ae

Department of Mathematics, Faculty of Sciences, University of Sharjah, Sharjah, United Arab Emirates 